\title{
ADERÊNCIA DE DIABÉTICOS AO TRATAMENTO MEDICAMENTOSO COM HIPOGLICEMIANTES ORAIS
}

\author{
Adherence of diabetics patient to pharmacological treatment \\ with oral hypoglycemic agents
Adhesión de diabéticos al tratamiento con hipoglucemiantes orales

Márcio Flávio Moura de Araújo ${ }^{1} \quad$ Ticiana da Cunha Gonçalves ${ }^{2} \quad$ Marta Maria Coelho Damasceno $^{3} \quad$ Joselany Áfio Caetano ${ }^{4}$

\section{RESUMO}

Objetivou-se identificar a adesão de diabéticos de Sobral - CE ao tratamento farmacológico com hipoglicemiantes orais. Investigaram-se 79 diabéticos de seis unidades básicas de saúde de Sobral - CE mediante visitas domiciliárias durante o período de março a junho de 2007. Para coleta de dados utilizou-se um formulário estruturado e a aplicação do Teste de Morisky e Green adaptado. Um pouco mais da metade dos estudados, $54,5 \%$, referiu não ter o cuidado de cumprir o horário de ingestão dos fármacos preestabelecido; a maioria não se esquece de tomar a medicação (66\%). Ademais, $90 \%$ dos investigados apresentam sentimento de pesar ao deixar de tomar os hipoglicemiantes orais. Identificou-se que 54,4\% e 45,5\% eram menos aderentes e mais aderentes à terapia medicamentosa com hipoglicemiantes orais, respectivamente. A adesão à terapia farmacológica com hipoglicemiantes orais é fundamental para um bom controle glicêmico e a prevenção de complicações micro e macrovasculares.

Palavras-chave: Diabetes melito. Cooperação do Paciente. Hipoglicêmicos.

\begin{abstract}
The objective was to identify the adherence of the diabetic from Sobral - CE, Brazil, to pharmacological treatment with oral hypoglycemic agents. Were investigated 79 diabetics from six basic health units in Sobral-CE, Brazil, through home visits carried out between March and June 2007. For data collection, a structured form was used and the adapted Morisky-Green test was applied. Slightly more than half of the subjects (54.5\%) indicated being careful don't to comply with the pre-established time for medication intake; most of them does not forget to take the medication (66\%). Moreover, $90 \%$ of the subjects regret forgetting to take the oral hypoglycemic agents. It was identified that $54.4 \%$ and $45.5 \%$ were less adherent and more adherent to the drug therapy with oral hypoglycemic agents, respectively. Adherence to pharmacological treatment with oral hypoglycemic agents is fundamental for good glucose control and to prevent micro and macrovascular complications.
\end{abstract}

Keywords: Diabetes mellitus. Patient compliance. Hypoglycemic Agents.

\section{Resumen}

El objetivo fue identificar la adhesión de diabéticos de Sobral - CE, Brasil, al tratamiento farmacológico con agentes hipoglucemiantes orales.Fueron investigados 79 diabéticos de seis unidades básicas de salud de Sobral-CE, Brasil, mediante visitas domiciliarias durante el período de marzo a junio de 2007. Para la recolecta de datos fue utilizado un cuestionario estructurado y la aplicación del Test de Morisky y Green adaptado. Poco más de la mitad de los estudiados, $54.5 \%$, dijo no tener cuidado de cumplir con el horario preestablecido para la ingestión de los fármacos; la mayoría no se olvida de tomar la medicación (66\%). Además, el $90 \%$ de los investigados presentó sentimientos de aflicción al dejar de tomar los hipoglucemiantes orales. Se identificó que el $54.4 \%$ y el $45.5 \%$ presentaban menos adherencia y mayor adherencia - respectivamente - a la terapia con fármacos hipoglucemiantes orales. .La adhesión a la terapia farmacológica con hipoglucemiantes orales es fundamental para un buen control glicémico y para la prevención de complicaciones micro y macrovasculares.

Palabras clave: Diabetes Mellitus. Cooperaçión del paciente. Agentes Hipoglucémicos

'Enfermeiro. Mestrando do Programa de Pós-graduação em Enfermagem da Universidade Federal do Ceará. Bolsista CAPES. Brasil. E-mail: marciofma@yahoo.com.br, ${ }^{2}$ Acadêmica do curso de Enfermagem da Universidade Vale do Acaraú. Bolsista PIBIC-CNPq. Brasil. E-mail: ticicunh@bol.com.br, ${ }^{3}$ Doutora em Enfermagem. Docente do Programa de Pós-graduação em Enfermagem da Universidade Federal do Ceará. Brasil. Email: martadamasceno@terra.com.br, ${ }^{4}$ Doutora em Enfermagem. Docente do Programa de Pós-graduação em Enfermagem da Universidade Federal do Ceará. Brasil. E-mail: joselany@ufc.br 


\section{INTRODUÇ̃̃}

Atualmente a prevalência do diabetes melito tipo 2 (DM 2) atinge proporções pandêmicas, demandando alto custo tanto econômico como social. Segundo estimativas da Organização Mundial de Saúde (OMS), o número de diabéticos no mundo em 2000 era de 177 milhões, com perspectiva de alcançar 350 milhões em 2025. No Brasil, em 2006, havia cerca de seis milhões de diabéticos, a expectativa é esse número chegar a 10 milhões em 2010. Outra característica epidemiológica do DM 2 é que ele vem crescendo vertiginosamente entre os países pobres e em desenvolvimento, contribuindo para o agravamento da pobreza nessas regiões. ${ }^{1,2}$

Nesse sentido, particular atenção tem sido dispensada aos diferentes níveis de prevenção do DM2. Sobretudo, a prevenção secundária, quando a patologia já existe, tem suscitado debates, haja vista que pesquisas têm demonstrado a importância do tratamento adequado do DM2 sem complicações para a promoção da saúde do diabético. ${ }^{2}$

Diante da gênese de complicações crônicas, a questão mais importante e desafiadora para os profissionais de saúde que cuidam de diabéticos é 0 controle da glicemia. Hoje, mediante estudos clínicos, controlados e randomizados, renomados, como o United Kingdom Prospective Diabetes Study (UKPDS), o Diabetes Control and Complications Trials (DCCT) e o Stockholm Diabetes Intervention Study (SDIS), ${ }^{3-5}$ está provado que o controle rigoroso da glicemia pode realmente reduzir o risco tanto para alterações microvasculares como para as macrovasculares características do DM2. ${ }^{3-6}$

Estudo sobre o impacto de programas educativos na qualidade do cuidado ao diabetes na América Latina constatou controle metabólico inadequado em $72 \%$ dos casos pesquisados. ${ }^{6} 0$ DM2 é um distúrbio metabólico progressivo, complexo e de difícil tratamento ao longo do tempo. De modo geral, a maior parte desses clientes está com excesso de peso, ou ainda é hipertensa. Desse modo, eles serão incapazes de conseguir se manter próximo de uma normoglicemia sem a adoção de antidiabéticos orais. ${ }^{5,7}$ Outro agravante diz respeito ao fato de muitos pacientes acreditarem ser dispensável a terapia farmacológica em virtude do caráter assintomático assumido pela doença em algumas situações. ${ }^{8}$

Atualmente é enorme 0 arsenal de fármacos adotados no estabelecimento de uma normoglicemia em diabéticos. Contudo, todo esse cenário se anula diante da não aderência desses clientes a essas drogas. Diante desta realidade, a adesão farmacológica de diabéticos coloca-se como uma temática relevante de saúde pública. ${ }^{5,9}$

Sob essa ótica, os métodos indiretos são considerados práticos e econômicos e, assim, têm sido propostos para medir o apego ao tratamento farmacológico, ou seja, verificar a concordância entre as orientações dos profissionais de saúde e o comportamento dos clientes. Entre eles destaca-se, mundialmente, o Teste de Morisky \& Green já adaptado para verificar a adesão de clientes asmáticos, hipertensos, portadores de artrite reumática entre outras patologias. ${ }^{10}$

Tendo em vista ser o tema relevante para a prática clínica de enfermagem, por ser o enfermeiro um dos principais profissionais engajados no cuidado desses clientes, e por ele poder fornecer subsídios para um cuidado integral ao cliente diabético, objetivou-se, por com este estudo, identificar a aderência de diabéticos do município de Sobral - CE, Brasil, ao tratamento medicamentoso com hipoglicemiantes orais.

\section{MATERIAL E METÓDOS}

Trata-se de um estudo seccional exploratório, desenvolvido em Unidades Básicas de Saúde da Família (UBASFs) da zona urbana do município de Sobral - CE e nos domicílios dos respectivos diabéticos estudados durante o período de março a junho de 2007. Inicialmente, a proposta era investigar as 15 UBASFs que constituíam um distrito referência do município; contudo, após convite oficial dos autores, somente seis UBASFs mostraram disponibilidade para ser sítio do estudo.

A opção pela área urbana decorreu da consistência dos indicadores de saúde das unidades circunscritas nessa zona, por serem mais organizados e detalhados, o que facilitava a localização dos diabéticos.

Para determinar a população base da pesquisa, foram coletados dados nos sistemas de informação de cada UBASF. Detectaram-se 103 diabéticos cadastrados nos seis estabelecimentos de saúde. Mediante esse 
quantitativo, foram aplicados os seguintes critérios de inclusão, a saber: 1. Ter comprovação clínica de diagnóstico de DM2 não insulinodependente; 2. Ter idade superior a 18 anos. 3. Estar cadastrado e em tratamento há pelo menos seis meses na respectiva UBASF; 4. Estar em tratamento com hipoglicemiante oral. De acordo com o estabelecimento dos critérios de inclusão, houve a exclusão de 24 sujeitos. Dessa forma, a amostra do estudo culminou em 79 diabéticos. Entretanto, não houve perdas de pesquisados quanto a hospitalizações, óbitos ou desligamento da respectiva UBASF.

A coleta de dados ocorreu mediante o registro de um formulário, previamente testado em estudopiloto anterior, durante entrevistas pré-agendadas pelos autores nos domicílios dos diabéticos. Além da questão da adesão ao tratamento farmacológico, nessa ocasião, houve aspectos sociodemográficos utilizando as variáveis idade, sexo, renda familiar, escolaridade, ocupação e etc. Os aspectos clínicos foram abordados utilizando as variáveis estado nutricional (IMC), pratica de atividade física e etilismo.

0 estado nutricional dos pesquisados foi verificado a partir do cálculo de Índice de Massa Corporal (IMC), por meio da fórmula [peso $(\mathrm{kg}) /$ altura $\left(\mathrm{m}^{2}\right)$ ], e interpretado a partir das preconizações da Organização Mundial de Saúde (OMS). 0 peso foi obtido em uma única tomada, com os indivíduos descalços e com roupas leves, utilizando-se uma balança portátil digital com capacidade de $120 \mathrm{~kg}$ e uma precisão de 0,1 kg. A estatura foi verificada em uma única tomada, sendo utilizada uma fita métrica com escala de $0,5 \mathrm{~cm}$. No intuito de assegurar a precisão da estatura, os pesquisados foram orientados a se posicionarem eretos e imóveis, com as mãos espalmadas sobre as coxas e com a cabeça ajustada ao plano de Frankfurt. ${ }^{11}$

Já a prática de atividade física foi pontuada, segundo referencial teórico, naqueles que se exercitavam ao menos três vezes por semana durante 30 minutos, enquanto que para prática do etilismo aceitou-se a simples ingestão de álcool, mesmo esporádica, como fator determinante para incluir esse comportamento. ${ }^{12}$

Especificamente, acerca do grau de adesão dos diabéticos ao tratamento farmacológico com hipoglicemiantes orais, adotou-se o Teste de Morisky e Green adaptado. A adaptação do referido teste consiste em realizar os seus questionamentos a partir das drogas e pacientes estudados. ${ }^{10}$ No caso de hipertensos, questiona-se o uso de anti-hipertensivos, de soropositivos o uso de antirretrovirais e etc. Neste estudo, os questionamentos abordaram diabéticos e 0 uso de hipoglicemiantes orais, respectivamente.

0 Teste de Morisky e Green é uma escala psicrométrica com quatro itens aos quais os sujeitos respondem de forma dicotômica, isto é, "sim/não" e envolve as seguintes indagações: 1) Você, alguma vez, se esquece de tomar os hipoglicemiantes orais?; 2) Você, às vezes, é descuidado quanto ao horário de tomar seu remédio?; 3) Quando você se sente bem, alguma vez deixa de tomar o remédio?; 4) Quando você se sente mal, alguma vez, você deixa de tomar o remédio? Um sim equivale a zero ponto, enquanto um não equivale a 1 ponto. Admite-se que o paciente é mais aderente ao tratamento, caso ocorra pelo menos 4 pontos; por sua vez, três ou menos pontos no teste indicam que o indivíduo é menos aderente ao tratamento farmacológico. ${ }^{10}$

Como exigido, o desenvolvimento do estudo transcorreu após o aval das UBASFs e aprovação da Comissão de Ética em Pesquisa da Universidade Estadual Vale do Acaraú sob o protocolo 193 em 21 de junho de 2007. Todos os pesquisados apresentaram o termo de consentimento assinado para participar do estudo.

\section{RESULTADOS}

0 estudo revelou que os pacientes pesquisados, eram, predominantemente, do sexo feminino $(87,3 \%)$. Em contrapartida, sobre a faixa etária, foi identificado que $27,8 \%, 28,8 \%$ e $30,6 \%$ tinham entre 61 e 70,51 e 60, e 71 e 90, respectivamente. Em virtude, talvez, da predominância da faixa senil entre os avaliados, grande parte deles está aposentada $(62,0 \%)$ ou restrita ao $\operatorname{lar}(29,2 \%)$. Além disso, tinham uma condição educacional e financeira deficitária, pois a maioria era analfabeta $(40,5 \%)$ ou tinha o ensino fundamental incompleto $(30,4 \%)$, e a grande maioria $(93,6 \%)$ vivia com uma renda familiar de menos de um a três salários mínimos mensais (Tabela 1). 
Tabela 1. Distribuição dos pesquisados, segundo dados sociodemográficos, estado nutricional, prática de atividade física e etilismo. Sobral - CE, 2007.

\begin{tabular}{|c|c|c|}
\hline Variáveis & $n$ & $\%$ \\
\hline \multicolumn{3}{|l|}{$\overline{\text { Sexo }}$} \\
\hline Masculino & 10 & 12,7 \\
\hline Feminino & 69 & 87,3 \\
\hline \multicolumn{3}{|l|}{ Idade } \\
\hline$\geq 18$ anos a 30 anos & 1 & 1,3 \\
\hline 31 a 40 anos & 2 & 2,7 \\
\hline 41 a 50 anos & 6 & 7,8 \\
\hline 51 a 60 anos & 23 & 28,8 \\
\hline 61 a 70 anos & 22 & 27,8 \\
\hline 71 a 90 anos & 25 & 30,6 \\
\hline \multicolumn{3}{|l|}{ Ocupação } \\
\hline Aposentado & 49 & 62,0 \\
\hline Do lar & 23 & 29,2 \\
\hline Autônomo & 3 & 3,8 \\
\hline Emprego formal & 2 & 2,5 \\
\hline Desempregado & 2 & 2,5 \\
\hline \multicolumn{3}{|l|}{ Escolaridade } \\
\hline Analfabeto & 32 & 40,5 \\
\hline Alfabetizado & 18 & 22,8 \\
\hline $\begin{array}{l}\text { Ensino fundamental } \\
\text { incompleto }\end{array}$ & 24 & 30,4 \\
\hline $\begin{array}{l}\text { Ensino fundamental } \\
\text { completo }\end{array}$ & 1 & 1,3 \\
\hline Ensino médio completo & 4 & 5,0 \\
\hline \multicolumn{3}{|l|}{ Renda mensal ${ }^{*}$} \\
\hline$\leq 1$ a 3 salários mínimos & 74 & 93,6 \\
\hline$>3$ até 5 salários & 4 & 5,1 \\
\hline$>5$ até 7 salários & 1 & 1,3 \\
\hline \multicolumn{3}{|l|}{ IMC } \\
\hline Normal & 16 & 20,2 \\
\hline Sobrepeso & 25 & 31,6 \\
\hline Obesidade I & 27 & 34,1 \\
\hline Obesidade II & 7 & 8,8 \\
\hline Obesidade mórbida & 4 & 5,3 \\
\hline \multicolumn{3}{|l|}{ Sedentarismo } \\
\hline Sim & 45 & 57,0 \\
\hline Não & 34 & 43,0 \\
\hline \multicolumn{3}{|l|}{ Etilismo } \\
\hline Sim & 2 & 2,5 \\
\hline Não & 77 & 97,5 \\
\hline
\end{tabular}

*Salário mínimo nacional de $\mathrm{R} \$$ 300, 00 reais em 2007.

Conforme mostram os dados, a maioria dos diabéticos estudados tinha obesidade do tipo I ou sobrepeso: $34,1 \%$ e $31,6 \%$, respectivamente. No que se refere à prática de atividade física, houve um equilíbrio, pois, enquanto $57 \%$ se enquadravam como sedentários, o restante (43\%) exercia atividades físicas regularmente. Consoante com os achados, constata-se que praticamente não havia etilistas no grupo investigado (Tabela 1). 
Tabela 2-Distribuição dos pesquisados, usuários de hipoglicemiantes orais, segundo respostas ao Teste de Morisky e Green. Sobral-CE, 2007.

\begin{tabular}{|c|c|c|}
\hline Variáveis & $\mathrm{N}$ & $\%$ \\
\hline \multicolumn{3}{|c|}{ Descuidado no horário da medicação } \\
\hline Sim & 43 & 54,5 \\
\hline Não & 36 & 45,5 \\
\hline \multicolumn{3}{|c|}{ Esquece de tomar seu medicamento } \\
\hline Sim & 27 & 34 \\
\hline Não & 52 & 66 \\
\hline \multicolumn{3}{|c|}{$\begin{array}{l}\text { Sente-se mal ao deixar de tomar o } \\
\text { medicamento }\end{array}$} \\
\hline Sim & 11 & 14 \\
\hline Não & 68 & 86 \\
\hline \multicolumn{3}{|c|}{$\begin{array}{l}\text { Sente-se bem ao deixar de tomar o } \\
\text { medicamento }\end{array}$} \\
\hline Sim & 8 & 10 \\
\hline Não & 71 & 90 \\
\hline \multicolumn{3}{|c|}{ Resultado do Teste de Morisky Green } \\
\hline Adere ao tratamento & 36 & 45,5 \\
\hline Não adere ao tratamento & 43 & 54,4 \\
\hline
\end{tabular}

Fonte: Dados da pesquisa de campo com um total de 79 pacientes.

Observou-se que a maioria dos estudados não se esquece de tomar a medicação (66\%). Ademais, entre os investigados predominou, de modo substancial $(90 \%)$, o sentimento de pesar ao deixar de tomar os hipoglicemiantes orais. Em contrapartida, $68 \%$ dos entrevistados afirmam não manifestar qualquer desconfor to psicológico ou emocional ao deixar de tomar a medicação. Um pouco mais da metade dos estudados (54,5\%) referiu ser descuidado com o cumprimento do horário de tomada dos fármacos hipoglicemiantes preestabelecidos. Por fim, em consonância com os dados da pesquisa, constatou-se haver um predomínio de sedentários, ainda que não expressivo entre os menos aderentes e os mais aderentes à terapia medicamentosa exclusiva com hipoglicemiantes orais: $54,4 \%$ e $45,5 \%$, respectivamente (Tabela 2).

\section{DISCUSSÃO}

0 presente estudo contemplou, essencialmente, mulheres idosas com excesso de peso e sedentárias. De acordo com determinados autores, o DM é uma patologia metabólica frequente com a maturidade em decorrência da queda da homeostase glicêmica. Por se tratar de idosos que, muitas vezes, estão com suas funções cognitivas comprometidas, o tratamento eficaz também fica comprometido caso as devidas medidas não sejam adotadas. Mesmo se tratando de usuários da rede pública, outro aspecto a ser considerado é o econômico, pois quase sempre o orçamento desses clientes é insuficiente para suprir os gastos com a terapêutica do DM como, por exemplo, alterações na dieta, pele, calçados e etc. ${ }^{6}$

No panorama global, a prevalência do DM 2, em 2000, foi de 2,3\%, mas de acordo com as projeções para 2030, esse valor será 4,4\%. ${ }^{13}$ Nos Estados Unidos, em 2003, cerca de $20 \%$ dos adultos norte-americanos com 65 anos ou mais eram acometidos por DM2, ou seja, aproximadamente, sete milhões de pessoas. Nesse sentido, ressalta-se, ainda, 0 seguinte: essa faixa etária, até 2010, terá um incremento de $12,6 \%$ para $13,2 \%$. Além disso, esses sujeitos terão outras complicações, especialmente a obesidade e a hipertensão arterial..$^{14}$

Nesse sentido, os enfermeiros devem fazer esforços para alcançar a meta de reduzir as condições mórbidas que predispõe ao surgimento de problemas cardiovasculares, como o é o caso da hipertensão e do DM2 $\cdot{ }^{15} \mathrm{~A}$ consulta de enfermagem e um processo de educação em saúde permanente são recursos importantes para o alcance da meta supracitada, já que podem repercutir em uma melhora do cumprimento da terapia medicamentosa.

Em relação à adesão farmacológica aos antidiabéticos orais, o Teste de Morisky e Green demonstrou que parte dos 
pesquisados eram menos aderentes ao tratamento farmacológico. A efetividade do tratamento de doenças crônicas depende, essencialmente, de dois fatores: a eficácia do tratamento prescrito e a adesão do paciente ao seu tratamento. Contudo, como mencionado anteriormente, muitas pessoas descumprem as recomendações. Entre as causas mais comuns de descumprimento incluem-se: não adquirir a medicação; tomar dose incorreta ou adequada, porém em intervalos incorretos; existir falha na comunicação profissional de saúde-paciente, entre outros. ${ }^{10}$

0 conceito de adesão varia entre diversos autores, mas, de forma geral, é compreendido como o uso dos medicamentos prescritos em pelo menos $80 \%$ do seu total, observando-se horários, dose e tempo de tratamento. ${ }^{16} \mathrm{~A}$ falta de adesão ao tratamento é algo cada vez mais destacado, especialmente entre os portadores de enfermidades crônicas, nas quais se insere o DM2. Conforme verificado por estudo denominado "Diabetes na América Latina", 78\% dos diabéticos avaliados apresentavam um controle glicêmico inadequado, caracterizado por glicemia venosa de jejum ${ }^{3} 110 \mathrm{mg} / \mathrm{dL}^{17}$

Também como verificado em pesquisa brasileira em um hospital de Ribeirão Preto, 32,2\% dos pacientes diabéticos não sabiam o nome da medicação utilizada; $51,6 \%$ tomavam o medicamento em horário inadequado, e a maioria tinha apenas noção do mecanismo de ação dos antidiabéticos orais prescritos. ${ }^{8}$ Outro estudo nacional realizado em Pelotas-RS constatou o seguinte: cerca de $40 \%$ dos pacientes em uso de insulina ou hipoglicemiantes orais apresentavam um controle glicêmico insatisfatório. 0 relato do uso incorreto dos medicamentos esteve associado a um aumento de $84 \%$ no risco de o paciente apresentar controle insatisfatório. ${ }^{18}$

De modo geral, o cumprimento do tratamento farmacológico representa uma complexa interação entre três pilares: fatores sociais, fatores relativos ao paciente e fatores relativos aos profissionais de saúde. Aspectos como condição socioeconômica e cultural, idade, sexo, estado civil (fatores sociodemográficos), tipo de fármaco prescrito, quantidade de comprimidos por dia (fatores relativos ao tratamento medicamentoso), tempo de doença, enfermidades e medicamentos associados (fatores clínicos) e orientações recebidas dos profissionais de saúde se relacionam com o êxito ou fracasso da adesão a regimes farmacológicos. ${ }^{19}$

Determinados autores veem o profissional de saúde como ponto central para a adesão farmacológica, como se ela fosse determinada exclusivamente pelo poder do profissional em persuadir seu cliente a seguir a prescrição. De qualquer forma, como evidenciado por estudos, a confiança depositada pelo paciente na prescrição, na equipe de saúde ou no profissional pessoalmente é fundamental na adesão farmacológica. ${ }^{5,9}$

Por serem clientes crônicos, acompanhados, continuamente, por profissionais de saúde, é imprescindivel que os diabéticos tenham respostas corretas para as próprias dúvidas acerca do manejo clínico da sua enfermidade. Isto, além de conquistar a credibilidade nesses profissionais, se traduzirá em um grau muito baixo de incerteza terapêutica, pois, na ausência ou má qualidade da informação, o cliente diabético não possui parâmetros para um autocuidado. ${ }^{20}$

Nesse sentido, uma investigação portuguesa sugere que quanto maior é o nível de incerteza em relação à doença em face do diagnóstico e tratamento, menos os diabéticos se sentem motivados para adotar um estilo de vida saudável, respeitando tratamento medicamentoso, controle glicêmico e prática de exercício físico. Conforme reitera o estudo, acerca do panorama terapêutico do DM2, a educação em saúde pode reduzir em $80 \%$ o manejo clínico inadequado dessa enfermidade metabólica. ${ }^{20}$

No transcurso da doença, o diabético enfrenta uma série de dificuldades como conflitos pessoais e sociais. Neste sentido, a abordagem do tratamento de enfermagem deve incluir o enfrentamento de uma ampla variedade de problemas, além da adesão farmacológica. Portanto, é crucial disponibilizar um cuidado individualizado, multiprofissional e de qualidade para esses clientes. Nesse intuito, as ações de educação em saúde são importantes, na medida em que se sensibilizam para a adesão farmacológica e para mudanças de comportamentos passíveis de promover a saúde do diabético.

Uma das limitações deste estudo reside no fato de o grau de adesão ter sido estudado apenas sob o ponto de vista do relato do diabético em relação ao horário de tomada dos medicamentos, em relação ao esquecimento e ao sentimento em deixar de tomar o medicamento. Isso difere de outras publicações que analisaram a adesão por meio ou do controle glicêmico, ${ }^{17}$ da análise de associação entre uso incorreto de medicamento e aumento no risco de o paciente apresentar controle insatisfatório, ${ }^{18}$ ou do conhecimento do paciente sobre o nome da medicação e do mecanismo de ação dos antidiabéticos orais. ${ }^{8}$ Dessa maneira, acredita-se ser importante o desenvolvimento de outros estudos com amostra e delineamento mais representativos. 


\section{CONSIDERAÇÕES FINAIS}

Apesar das limitações do estudo, particularmente por ter sido desenvolvido com apenas seis UBASFs, o que impede a realização de inferências populacionais, alguns aspectos positivos podem ser ressaltados. Na dinâmica da sua doença, 0 diabético requer não apenas cuidados com a aderência medicamentosa, mas também orientações em saúde quanto a dieta, prática de atividade física, redução do estresse, entre outras.

Diante do observado, acredita-se ser prioritário, para os enfermeiros desse município e de outras localidades nacionais, o desenvolvimento de investigações mais bem delimitadas e com população maior, com a finalidade de se obter um quadro mais fidedigno dessa problemática. Uma proposta plausível seria a adoção de Teste Morisky e Green que se mostrou prático na delimitação de diabéticos menos aderentes ao tratamento farmacológico. Fundamentados nas devidas informações, será possível estabelecer medidas interventivas de impacto na promoção da saúde dos diabéticos usuários de hipoglicemiantes orais.

\section{REFERÊNCIAS}

1. Ministério da Saúde (BR). Caderno de Atenção Básica, 16. Normas e manuais técnicos. Brasília (DF); 2006.

2. Sociedade Brasileira de Diabetes. Atualização sobre diabetes. Rio de Janeiro (RJ); 2006.

3. United Kingdom Prospective Diabetes Study Group-UKPDS. Intensive blood-glucose control with sulphonylureas or insulin compared with conventional treatment and risk of complications in patients with type 2 diabetes (UKPDS33). Lancet 1998 0ct; 352(31): 837-53.

4. Diabetes Control and Complications Trial Research Group-DCCT. The effect of intensive treatment of diabetes on the development and progression of long-term complication in insulin dependent diabetes mellitus. N England J Med 1993 Sep; 329(14): 977-86.

5. Skyler JA. Relação do controle glicêmico com as complicações diabeticas. In: Inzucchi S. Diabete Melito: manual de cuidados essenciais. Porto Alegre (RS): Artmed; 2007. p.334-47.

6. Gagliardino JJ, De la Hera M, Siri F. Grupo de investigación de la rede QUALIDIAB-evaluación de la calidad de la assistencia al paciente diabético em América Latina. Rev Panam Salud Publica. 2001 nov; 10(5): 309-17.

7. Krentz AJ, Bailey CJ. Oral antidiabetic agents- current role in type 2 diabetes mellitus Drugs. 2005; 65(3): 385-411.

8. Gimenes HT, Zanetti ML, Otero LM, Teixeira CRS. 0 conhecimento do paciente diabético tipo 2 acerca dos antidiabéticos orais. Cienc \& Cuid Saude 2006 set/dez; 5(3): 317-25.
9. Grant R, Adams AS, Trinacty CM, Zang F, Kleinman K, Soumerai SB, et al. Relationship between patient medication adherence and subsequent clinical inertia in type 2 diabetes glycemic management. Diabetes Care 2007 Apr; 30(4): 807-12.

10. Morisk DE, Green LW, Levine DM. Concurrent and predictive validity of a self-reported measure of medication adherence. Med Care 1986 Jan; 24(1): 67-73.

11. Organización Mundial de la Salud- OMS. El estado físico: uso e interpretación de la antropometria: informe de un Comité de Expertos de la OMS. Ginebra; 1995.

12. World Health Organization. Obesity: preventing and managing the global epidemic. Geneva; 2000.

13. Wild S, Roglic G, Green A, Sicree R, King H. Global prevalence of diabetes- estimates for the year 2000 and projections for 2030 . Diabetes Care 2004 May; 27(5):1047-053.

14. Balkrishan R, Rukmini R, Camacho FT, Huston AS et al. Predictors of medication adherence and associated health care costs in an older population with type 2 diabetes mellitus: a longitudinal cohort study. Clin Ther 2003 Nov; 25(11): 2958-971.

15. Assis LS, Stipp MAC, Leite JL, Cunha NM. A atenção da enfermeira à saúde cardiovascular de mulheres hipertensas. Esc Anna Nery Rev Enferm 2009 abr/jun; 13(2): 265-70.

16. Leite SN, Vasconcelos MPC. Adesão a terapêutica medicamentosa: elementos para discussão de pressupostos adotados na literatura. Cienc \& Saude Colet 2003 mar/abr; 8(3):775-82.

17. Stewart GL, Tambascia M, Guzmán JR, Etchegoyen F et al. Control of type 2 diabetes in private practice in nine countries of Latin America. Rev Panam Salud Publica 2007 jul; 22(1): 12-20.

18. Araújo RB, Santos I, Cavaleti MA, Costa JSD, Beria JU. Avaliação do cuidado prestado a pacientes diabéticos em nível primário. Rev Saude Publica. 1999 fev; 33(1): 24-32.

19. Kidd KE, Altman DG. Adherence in social context. Control Clin Trials 2000 0ct; 21(5 suppl 1): S184-187.

20. Apóstolo JLA, Viveiros CSC, Nunes HIR, Domingues HRF. Incerteza na doença e motivação para o tratamento em diabéticos tipo 2. Rev Latino-am Enfermagem 2007 jul/ago; 15(4): 575-82.
Data de recebimento: 12/03/2009 Data de reapresentação: 04/19/2009 Data de aprovação: 18/11/2009 